\title{
Abdominal angiostrongyliasis mimicking acute appendicitis
}

A 57-year-old Guatemalan man with no relevant medical or surgical history presented with an acute onset of sharp, non-radiating lower abdominal pain of 12 hours duration. His social history included smoking a daily pack of cigarettes and working as a fisherman on the tropical Guatemalan south coast. The patient reported fevers up to $101^{\circ} \mathrm{F}$, nausea, and three episodes of nonbloody vomiting in the preceding 12 hours. He stated that 3 hours prior to presentation, the pain had become generalized over his entire abdomen.

On admission, the patient was in acute distress because of the pain and was febrile $\left(102^{\circ} \mathrm{F}\right)$. Abdominal examination revealed that his abdomen was tense, diffusely tender to palpation, with nonvoluntary guarding, marked rebound tenderness, and absent bowel sounds. His white blood cell count was raised at $13 \times 10^{9} / \mathrm{L}$ with $26 \%$ eosinophils, but his serum electrolyte levels were normal, and stool examination was negative for ova and parasites. An initial abdominal plain radiograph revealed subdiaphragmatic gas.

The patient was taken to the operating room for an exploratory laparotomy. Intraoperative findings included abundant amounts of intestinal contents in the peritoneal cavity, an intact appendix with no inflammatory changes, partial necrosis of the terminal ileum $2 \mathrm{~cm}$ from the ileocecal valve, and necrosis with perforation of the cecum. He underwent peritoneal lavage, appendectomy, resection of $15 \mathrm{~cm}$ of ascending colon ( $\bullet$ Fig. $1 \mathrm{a}$ ), and resection of $5 \mathrm{~cm}$ of distal ileum with an end-toend anastomosis. The patient had a satisfactory postoperative course and was discharged home.

Histologic evaluation of the specimens revealed dense eosinophilic infiltration in the intestinal tissue with granuloma formation and giant cells ( $\bullet$ Fig. 1 b), parasitic larvae with surrounding inflammation $(\bullet$ Fig. $1 \mathrm{c}$ ), and intravascular parasites causing thrombosis ( $\bullet$ Fig. 1 d). Findings were consistent with infection by Angiostrongylus costaricensis causing mesenteric ischemia and subsequent perforation. Abdominal angiostrongyliasis is a zoonotic disease caused by invasion of the mesenteric vessels by the nematode
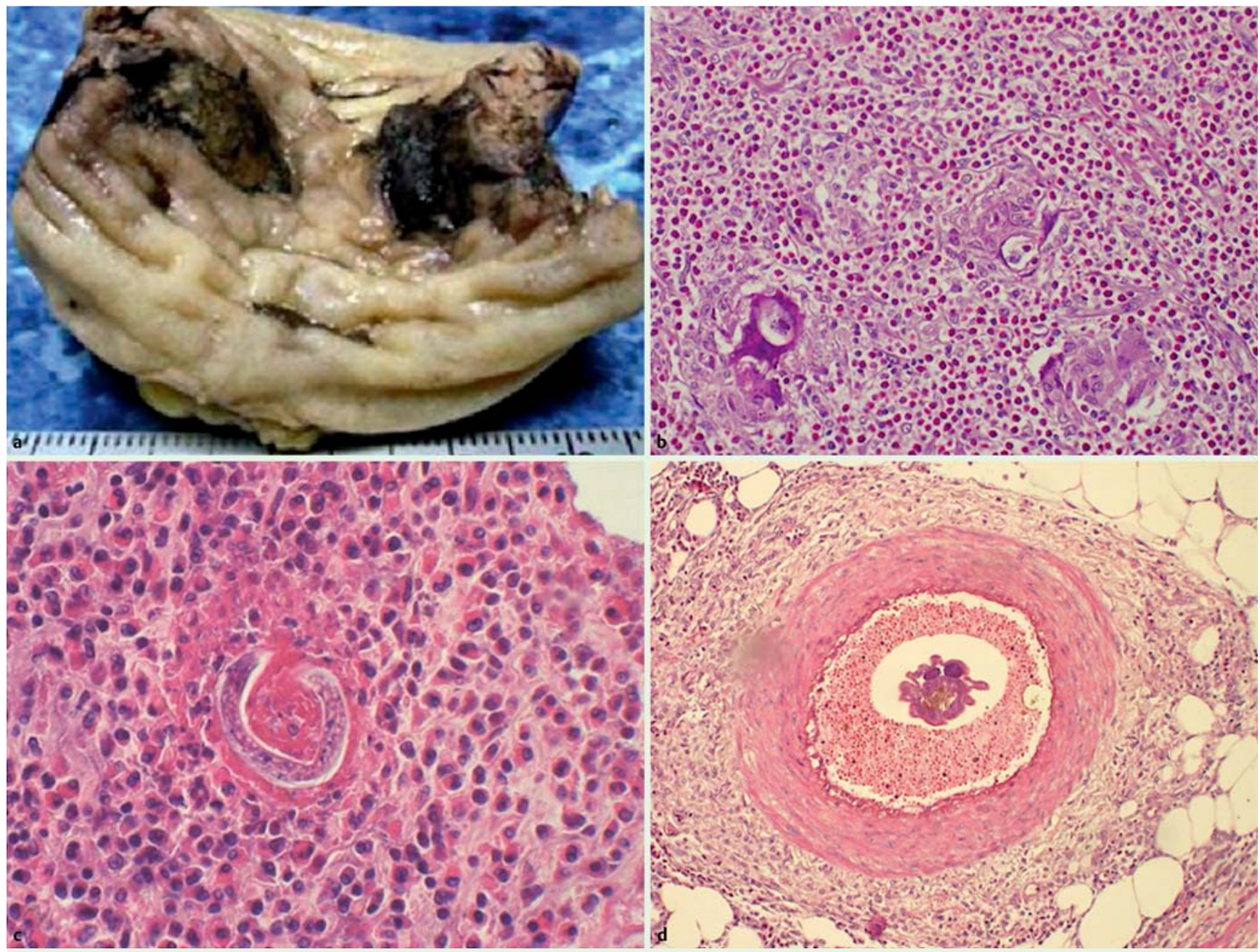

Fig. 1 Resected surgical specimen in a 57-year-old Guatemalan man who presented with bowel perforation. a The macroscopic appearance of the perforated cecum. $\mathbf{b}, \mathbf{c}, \mathbf{d}$ The microscopic appearance showing: $\mathbf{b}$ the granulomatous reaction in response to presence of parasite eggs; $\mathbf{c}$ tissue-invading larvae surrounded by a dense eosinophilic infiltrate characteristic of Angiostrongylus costaricensis; $\mathbf{d}$ intravascular parasites in a mesenteric vessel causing mesenteric vessel occlusion. 
Angiostrongylus costaricensis, whose definite and intermediate hosts are the rodent and slug, respectively. It is endemic to Latin America, with its prevalence inversely related to hygiene conditions [1]. Humans are accidental hosts, developing classical findings for acute appendicitis when mesenteric vessels are occluded by the intravascular parasite ( $\bullet$ Fig. 1d), and even bowel perforation [2]. No medical therapy has been identified for Angiostrongylus costaricensis. The complications, such as those seen in our patient, are treated surgically.

Endoscopy_UCTN_Code_CCL_1AD_2AZ

\section{Paul T. Kröner ${ }^{1}$, Victor Argueta ${ }^{2}$}

${ }^{1}$ Department of Medicine, Universidad Francisco Marroquín, Guatemala City, Guatemala

2 Department of Pathology, San Juan de Dios General Hospital, Guatemala City, Guatemala

\section{References}

1 Argueta VL. Angiostrongylus costaricensis: Reporte de cinco casos de Guatemala. Rev Latinoam Patol 2001; 39: 43-47

2 Kramer MH, Greer GJ, Quiñonez JF et al. First reported outbreak of abdominal angiostrongyliasis. Clin Infect Dis 1998; 26: $365-$ 372

\section{Bibliography}

Dol http://dx.doi.org/

10.1055/s-0034-1391501

Endoscopy 2015; 47: E179-E180

(c) Georg Thieme Verlag KG

Stuttgart · New York

ISSN 0013-726X

\section{Corresponding author}

\section{Paul T. Kröner, MD}

PGY-1

Mt. Sinai St. Luke's/Roosevelt Hospital Center Department of Internal Medicine

515 W 59th Street Apt. 15R.

New York City

NY, 10019

USA

PFlorit@chpnet.org

Competing interests: None 